\title{
Régulation et concurrence dans le transport collectif urbain
}

Aurélie Coppe et Axel Gautier*

\begin{abstract}
In Europe, most of the urban public transport services are not operated on a commercial basis. The implication of local public authorities in the sector is important : they organize their local market, they finance services and, sometimes, they also act as producers. This paper analyzes the public transport system in four European cities : Lyon, Stockholm, Helsinki and Brussels and concentrates, in particular, on the introduction of regulated competition. Four different market mechanisms can be used in the public transport sector : (1) competitive tendering, (2) open access with on-track competition, (3) yardstick competition where the operator's reward is based on a comparative evaluation of its performance and (4) the possibility of challenging the incumbent public operator. The pros and cons of different competitive mechanisms are presented and the article discusses the conditions under which a truly competitive market could be settled.
\end{abstract}

Keywords - public transport, regulation, competition.

En Europe, la toute grande majorité des services de transports collectifs urbains ne sont pas opérés sur une base commerciale. Les autorités publiques sont fortement impliquées dans l'organisation, la gestion et le financement du secteur. Dans cet article, nous analysons, au travers de l'expérience de quatre villes (Lyon, Stockholm, Helsinki et Bruxelles), les modalités d'introduction de la concurrence régulée dans le secteur du transport urbain.

\section{INTRODUCTION}

En Europe, la mobilité et le développement du transport collectif en zone urbaine sont deux enjeux importants. Dès lors, l'implication des autorités publiques dans le transport urbain est essentielle pour garantir la fourniture d'un service de qualité à des tarifs abordables. La gestion d'un réseau de transport urbain de qualité

Aurélie COPPE est chercheur aux Facultés universitaires Saint-Louis à Bruxelles.

Axel GAUTIER est chercheur aux Facultés universitaires Saint-Louis. II remercie la Région de Bruxelles-Capitale pour son soutien financier. Le contenu de l'article n'engage que les auteurs. gautier@fusl.ac.be 
représente une importante mission de service public (MSP) pour la collectivité, qui justifie l'octroi de subsides aux compagnies de transport.

En effet, la plupart des réseaux de transport urbain ne sont pas commercialement viables. La collectivité doit donc prendre en charge, en tout ou en partie, les coûts liés aux investissements en infrastructure et matériel roulant. Les opérateurs reçoivent également des subsides d'exploitation, qui diffèrent par leur importance selon les communautés. Par exemple, en 2002, les subsides couvraient $45 \%$ des coûts opérationnels à Helsinki, 50 \% à Stockholm, 51 \% à Lyon et 60 \% à Bruxelles.

Cependant, le rôle des autorités organisatrices du transport $(A O)$ ne se limite pas au paiement de subsides. Elles sont également responsables de la régulation et de l'organisation du secteur. La gestion d'un réseau de transport public requiert trois types de responsabilité : stratégique, tactique et opérationnelle. La responsabilité stratégique concerne le choix d'une politique de transport: les objectifs à atteindre en termes de parts de marché et de profitabilité, la sélection et la régulation des opérateurs, la définition des MSP et les moyens alloués pour le financement. La responsabilité tactique consiste à déterminer les caractéristiques du service nécessaires en vue d'atteindre les objectifs stratégiques : choix des fréquences, du système d'horaire, des tarifs et de la flotte de véhicules. Enfin, la responsabilité opérationnelle concerne la production et la vente de services de transport. Les AO sont en charge du niveau stratégique ; le niveau tactique est conservé par l'AO (Lyon, Helsinki, Stockholm) ou délégué à un opérateur (Bruxelles). Les responsabilités opérationnelles sont, quant à elles, confiées à un opérateur public (la STIB à Bruxelles), à un opérateur privé (la STLC à Lyon), à plusieurs opérateurs privés (Stockholm) ou, enfin, à plusieurs opérateurs privés et publics (Helsinki).

\section{LA RÉGULATION DES OPÉRATEURS}

Une fois les prestations de chaque opérateur définies, les opérateurs et l'AO doivent s'entendre sur le financement de celles-ci. Plusieurs solutions contractuelles sont possibles. Elles diffèrent quant au partage du risque commercial (sur les recettes) et du risque opérationnel (sur les coûts). Dénotons par $\mathrm{R}$ et $\mathrm{C}$ les recettes et les coûts attendus du trafic. Le service n'est pas commercialement viable si $\mathrm{R}<\mathrm{C}$. Pour que l'opérateur assure le service, l'AO doit, donc, lui transférer un montant $T$ égal à la différence $\mathrm{C}-\mathrm{R}>0$, augmenté d'une prime $\mathrm{U}$. Dénotons par $\Delta R=r-R$ la différence entre les recettes réalisées $(r)$ et les recettes attendues $(R)$ et par $\Delta \mathrm{C}=\mathrm{C}-\mathrm{C}$ la différence entre les coûts réalisés (c) et les coûts attendus $(\mathrm{C})$. Un contrat de régulation définit (1) la part $\alpha$ du risque commercial $\Delta R$ et (2) la part $\beta$ du risque opérationnel $\Delta \mathrm{C}$ prises en charge par l'AO. Suivant ce schéma, l'AO transfère à l'opérateur $T=U+C-R-\alpha \Delta R+\beta \Delta C$ et le profit de l'opérateur est de $\Pi=(1-\alpha) \Delta R-(1-\beta) \Delta C+U$. Si par exemple $\alpha=1$ et $\beta=0$, l'AO collecte les recettes du trafic $(T=U+C-r)$ et l'exploitant supporte le risque opérationnel. Ce contrat de régulation est de type gestion à prix forfaitaire. 
Au plus l'opérateur prend une part importante des risques à sa charge, au plus son profit est incertain. II exigera dès lors une prime de risque $U$ plus importante pour assurer les services. Par contre, si $\Delta \mathrm{R}$ et $\Delta \mathrm{C}$ dépendent de l'effort ${ }^{1}$ de l'opérateur, confier à celui-ci une part importante des risques est un moyen de l'inciter à fournir cet effort. A contrario, dans un contrat où l'opérateur ne supporte aucun risque, son profit est garanti et il n'a dès lors aucun incitant à augmenter ses recettes et/ou à réduire ses coûts. Le contrat de régulation doit tenir compte de ce lien entre partage des risques et incitation à l'effort ${ }^{2}$. Gagnepain (1998) observe que les opérateurs réglementés par un schéma moins incitatif ont des coûts plus élevés ${ }^{3}$.

L'AO n'est pas seulement concernée par le coût du service mais également par la qualité de celui-ci. Dans le cas du transport urbain, la qualité du service se mesure à plusieurs critères : fréquence, ponctualité, desserte, capacité, propreté, respect de l'environnement... Dans les quatre villes étudiées dans cet article, les contrats de régulation incluent, en plus du partage des risques, des dispositifs visant à inciter l'opérateur à fournir un service de qualité.

\section{L'INTRODUCTION DE MÉCANISMES CONCURRENTIELS}

L'introduction de mécanismes de marché a pour objectif la réduction des coûts opérationnels et/ou l'accroissement de la qualité du service. La concurrence ne supprime pas la nécessité de réguler le secteur ${ }^{4}$. D'après la Commission européenne ${ }^{5}$, les villes ayant introduit de la concurrence régulée dans le transport urbain ont un taux de croissance du nombre de passagers supérieur $(+3,1 \%)$ à celui des villes n'ayant pas introduit de concurrence $(-0,7 \%)$ et à celui des villes ayant introduit de la concurrence sans l'accompagner d'une régulation significative $(-3,1 \%)$. De même, le taux de couverture des coûts par les recettes du trafic augmente plus rapidement dans les villes utilisant la concurrence régulée (+ 1,7\%) que dans les villes où il n'y a pas de concurrence (+ 0,3\%) ou pas de régulation (+ 0,3 \%). À Stockholm, dans les années 90, le besoin de financement public pour le transport urbain a diminué de plus de 110 millions d'euros par an suite à l'introduction de la concurrence. Durant cette même période, l'offre de transport public a augmenté de plus de $20 \% 6$.

1. Dans la théorie de l'agence, le terme effort désigne l'ensemble des actions de l'opérateur ayant pour but soit d'augmenter les recettes du trafic soit de diminuer les coûts d'exploitation.

2. Laffont \& Tirole (1986).

3. Gagnepain \& Ivaldi (2002) estiment les gains d'efficacité associés à l'utilisation d'un contrat de régulation optimal.

4. En Angleterre, l'industrie du bus est dérégulée depuis 1985 et les compagnies peuvent librement décider de l'offre de services. La dérégulation a augmenté la productivité des entreprises mais l'utilisation du transport public a décliné en dépit d'une hausse de l'offre de services (Nash, 1993).

5. Commission européenne, COM (2002) 107.

6. EMTA News $n^{\circ} 14$, septembre 2003. 
Quatre mécanismes de marché peuvent être introduits dans le secteur du transport urbain :

1. L'appel d'offres : l'exploitation du réseau ou d'une partie de celui-ci est concédée à un (ou plusieurs) opérateur(s) par une procédure de soumission concurrentielle. Dans ce cas, les opérateurs sont en concurrence non pas sur le marché mais pour l'obtention du marché. L'AO sélectionne les opérateurs et leur rend l'infrastructure accessible. La sélection des opérateurs se fait sur la base de critères financiers (subsides demandés) et qualitatifs. Généralement, l'entreprise sélectionnée se voit octroyer un droit d'exploitation exclusif pour une durée déterminée. Les opérateurs sélectionnés doivent se conformer au cahier des charges qui prévoit l'offre à fournir et le financement de celle-ci. Une procédure d'appel d'offres n'est réellement concurrentielle que si l'adjudicateur traite l'ensemble des participants de manière équitable. Les autorités de la concurrence doivent veiller au bon fonctionnement du mécanisme.

2. La concurrence sur le réseau : le régulateur autorise la libre entrée sur le marché. Dans ce cas, soit plusieurs compagnies fournissent des services en parallèle - c'est le cas à Bruxelles où trois opérateurs de bus (STIB, TEC et DE LIJN) sont présents et ce, parfois sur des tronçons identiques ; soit il n'y a qu'un seul opérateur mais le marché est contestable. Pour cela, il faut que l'exploitation du service nécessite peu d'actifs spécifiques, ce qui est le cas pour les services de bus. L'expérience de dérégulation britannique montre cependant que la libre entrée ne garantit pas que le marché devienne concurrentiel ${ }^{7}$. La concurrence sur le réseau peut s'accompagner de règles communes édictées par l'AO comme l'utilisation d'un système d'horaires et de tarifs intégrés.

3. La gestion directe challengée : lorsqu'un opérateur reçoit un droit exclusif, les concurrents peuvent contester la position de l'opérateur implanté avec, comme argument, le fait qu'ils pourraient opérer un service comparable à un coût inférieur. L'opérateur fait face à une concurrence potentielle sur le marché rendu contestable.

4. Le benchmarking : l'AO rémunère les opérateurs sur la base des coûts d'un opérateur type du secteur. Si les coûts de l'opérateur sont plus élevés que le coût standard, le subside n'est pas affecté. Ce mécanisme de concurrence par comparaison incite fortement les opérateurs à contrôler leurs coûts. II est utilisé depuis la fin des années 80 dans plusieurs régions de Norvège et a eu un effet significatif sur les coûts opérationnels des compagnies ${ }^{8}$.

7. Pour Mackie, Preston \& Nash (1995), des barrières à l'entrée ont subsisté après la dérégulation comme la présence de coûts irrécupérables ("sunk costs »), celle d'économies dues à l'expérience et la possibilité pour les opérateurs en place de réagir rapidement à toute nouvelle entrée, celle-ci devant être préalablement notifiée.

8. Dalen \& Gomez-Lobo (2003). 


\section{RÈGLEMENTS EUROPÉENS}

La Commission européenne veut mettre en place un nouveau cadre réglementaire commun pour le transport urbain. Pour cela, elle a déposé en février 2002 au Parlement européen une deuxième version de son projet de règlement (COM (2002) 107). On y retrouve les quatre mécanismes concurrentiels décrits ci-dessus. La proposition prévoit deux schémas organisationnels pour le secteur. Le premier permet à l'AO d'accorder un droit d'exploitation exclusif à un opérateur pour autant que l'AO et l'opérateur signent un contrat de service public. La seconde permet d'accompagner la libre entrée sur le marché de règles communes à tous les opérateurs. À côté du projet de règlement, la Cour de Justice des Communautés Européennes (CJCE) vient de préciser les règles que les $A O$ doivent appliquer pour le financement des MSP. La CJCE recommande que les AO utilisent soit l'appel d'offres soit le benchmarking.

\subsection{Le contrat de service public}

Un contrat de service public est conclu entre une AO et un prestataire de services. Il prévoit le versement de subsides en contrepartie des MSP assurées par l'opérateur. Le texte de la Commission réglemente le contenu et l'attribution de ces contrats. En particulier, il prévoit que :

1. Le contrat est obligatoire pour toute entreprise qui reçoit un droit d'opération exclusif.

2. Le contrat est conclu pour une durée déterminée maximale de 8 ans pour les services de bus et de 15 ans pour les autres modes de transport avec cependant la possibilité d'étendre la durée du contrat pour tenir compte de la durée de vie des actifs si l'opérateur doit effectuer des investissements dans du matériel roulant spécifique.

3. L'attribution du contrat se fait normalement via un processus d'appel d'offres.

4. Des exceptions sont prévues pour les services de tram et de métro mais pas pour les services de bus.

5. Lorsqu'un contrat est attribué directement (sans appel d'offres), il peut être contesté par un opérateur alternatif.

Lorsqu'une AO choisit le contrat de service public, elle est tenue d'utiliser soit l'appel d'offres soit la gestion directe challengée, sauf pour les services de bus où l'appel d'offres est normalement obligatoire. Cette dernière disposition prive les AO de la possibilité d'organiser directement les services de bus. Elle fait l'objet de nombreuses critiques, notamment d'opérateurs qui se sont exprimés en faveur de la gestion directe challengée comme alternative à l'appel d'offres pour l'ensemble des services ${ }^{9}$.

9. Le groupe des grandes métropoles (GGM) a émis un avis dans ce sens en 2003. 


\subsection{Les règles communes}

Plutôt qu'un contrat de service public, les AO peuvent définir des règles communes, applicables sans discrimination à tous les opérateurs de leur zone géographique. Ces obligations ont par exemple trait au matériel roulant (technologie, aspect extérieur), à la participation à des systèmes intégrés d'horaire, de billetterie et d'information et à des obligations tarifaires. Une compensation pour la mise en conformité avec ces règles peut être accordée, pour autant qu'elle puisse être obtenue de manière non discriminatoire par tous les opérateurs et qu'elle reflète les coûts de mise en conformité.

\subsection{Le cas Magdeburg}

Ce cas, jugé le 24/7/2003 (JO 20/9/2003), opposait un opérateur privé évincé (Altmark Trans) à la ville de Magdeburg (Allemagne). La CJCE a dû se prononcer sur la possibilité pour les AO allemandes d'accorder des subsides à une compagnie de transport. L'arrêt de la CJCE précise que des subsides aux entreprises de transport urbain ne peuvent être accordés qu'aux conditions suivantes :

1. Les subsides couvrent des MSP clairement identifiées.

2. Les paramètres sur la base desquels les subsides sont calculés sont clairement spécifiés à l'avance.

3. La compensation ne peut excéder les coûts additionnels que l'entreprise doit supporter pour s'acquitter des MSP auxquels s'ajoute un profit raisonnable.

4. Si le prestataire de service n'est pas choisi par une procédure d'appel d'offres, les compensations doivent être calculées sur la base des coûts d'un opérateur type du secteur.

Si l'une de ces conditions n'est pas satisfaite, la CJCE considère que les subsides sont en partie des aides d'État et donc proscrits par les règles communautaires en matière de concurrence. La CJCE oblige les AO qui n'adoptent pas une procédure de soumission concurrentielle pour l'attribution des contrats de service public à pratiquer une évaluation comparative des coûts des MSP (benchmarking) afin d'éviter que d'éventuelles aides d'État ne soient versées au titre de compensation pour les MSP.

Actuellement, faute de position commune au sein des instances européennes, une certaine incertitude subsiste quant au futur cadre réglementaire. II est acquis que l'Europe veut introduire plus de concurrence dans le secteur - le type de concurrence à mettre en place faisant toujours l'objet de débats. Cependant, un grand nombre de municipalités européennes utilisent déjà des mécanismes de marché. Dans la section suivante, nous décrivons l'expérience de quatre villes en mettant en avant les avantages et les inconvénients des différents mécanismes concurrentiels. 


\section{L'INTRODUCTION DE LA CONCURRENCE DANS QUATRE VILLES EUROPÉENNES}

\subsection{Lyon}

Le réseau de transport en commun lyonnais est organisé et géré par le SYTRAL (le Syndicat mixte des Transports pour le Rhône et l'Agglomération lyonnaise) et la SLTC (la Société Lyonnaise des Transports en Commun). Le SYTRAL est l'AO du transport urbain et la SLTC, société privée filiale de KEOLIS, exploite actuellement le réseau. Les principales missions du SYTRAL consistent à élaborer et mettre en œuvre la politique de transport de l'agglomération lyonnaise, à réaliser les investissements nécessaires dans l'infrastructure et le matériel roulant et à déterminer la politique tarifaire et les adaptations de l'offre de transport. Le SYTRAL confie à la SLTC la gestion de l'intégralité du réseau dans le cadre d'un contrat de délégation de service public.

Le contrat liant le SYTRAL à la SLTC est une convention de gestion à prix forfaitaire d'une durée de 6 ans assortie d'un système d'intéressement basé sur les résultats de fréquentation, les recettes tarifaires, les coûts d'exploitation et la qualité de service. Le contrat actuel arrive à échéance le 31/12/2004. Un nouveau contrat de délégation de service public prendra effet le 01/01/2005. L'attribution par appel d'offres du contrat de gérance lyonnais est, de par sa complexité, un processus relativement coûteux. La première étape de la procédure de mise en concurrence pour la gestion du réseau consiste à choisir, parmi les entreprises candidates, celles qui seront autorisées à remettre une offre. Trois entreprises candidates ont reçu une autorisation : KEOLIS, TRANSDEV-RATP et CONNEX. Seules KEOLIS et TRANSDEV ont remis une offre. Le nouvel exploitant sera sélectionné mi-novembre. Les deux entreprises semblent se disputer âprement le marché.

La sélection du nouvel exploitant du réseau lyonnais illustre bien le problème que pose le maintien d'une concurrence effective dans le secteur. Cette concurrence est mise à mal par les éléments suivants : premièrement, l'exploitation d'un réseau de la taille et de la complexité de celui de Lyon nécessite des compétences techniques et managériales que seules quelques entreprises possèdent. II existe donc d'importantes barrières à l'entrée sur ce marché. Deuxièmement, le marché français est fortement concentré. En 2003, sur les 219 contrats de transport urbain répertoriés dans l'enquête CERTU/GART comprenant presque tous les réseaux français, à l'exception de l'île-de-France, 68,4 \% étaient confiés aux trois plus grands groupes de transport français (KEOLIS 28,3 \%, TRANSDEV 17,8 \% et CONNEX 22,3\%). Finalement, on observe rarement l'éviction de l'opérateur en place. Pour ces raisons, on peut légitimement craindre qu'en France, on assiste à une monopolisation des marchés locaux, la situation actuelle étant favorable à la mise en place de mécanismes de collusion tacite dans les procédures d'appels d'offres ${ }^{10}$.

10. Ivalidi et al. (2003). 


\subsection{Stockholm}

À Stockholm, la compagnie publique SL est responsable du transport public. Avant 1993, SL opérait l'ensemble des services de transport. Depuis, elle a progressivement délaissé sa tâche d'opérateur pour ne conserver que les responsabilités stratégiques et tactiques. SL recourt dès lors à la sous-traitance pour la fourniture de services de transport. Les opérateurs sont sélectionnés par une soumission concurrentielle. Actuellement, tous modes de transport confondus, six sous-traitants opèrent des services avec SL via un contrat de type gestion forfaitaire avec un système de bonus/pénalités lié à la qualité du service.

La présence de plusieurs opérateurs nécessite une coordination importante, mise en œuvre et gérée par SL. Elle définit un système de tarifs et horaires intégrés, gère la promotion et coordonne, au sein d'une de ses filiales, un service consommateur intégré. SL procure l'infrastructure et planifie les services ainsi que les investissements en infrastructure et en matériel roulant.

Le recours systématique à la sous-traitance, la concurrence pour l'octroi des contrats ainsi qu'une hausse du prix du ticket ont permis d'augmenter le taux de couverture des coûts par les recettes du trafic. En 1990, 70 \% des coûts opérationnels étaient financés par les subsides ; ce taux est passé à 50 \% en $2002^{11}$.

Le recours à plusieurs exploitants est un moyen de prévenir une trop grande concentration. Le marché reste cependant fort concentré : les six contrats de soustraitance sont attribués à cinq opérateurs ${ }^{12}$ de taille conséquente et les petits opérateurs ont disparu.

\subsection{Helsinki}

En 1998, la ville d'Helsinki a scindé la société HKL, son AO qui opérait des services de transport public, en deux entités : I'une organisationnelle et l'autre opérationnelle. $\mathrm{HKL}$ opère également des services de bus dans la région métropolitaine d'Helsinki pour le compte d'une autre AO, YTV. À l'inverse de SL, HKL garde une responsabilité opérationnelle. Ceci permet d'éviter la création d'un monopole et permet un benchmarking aisé sur la base des coûts de l'opérateur public.

À Helsinki, le transport par bus est donc assuré par des opérateurs publics (2) et privés, choisis sur la base d'appels d'offres. Les contrats de délégation, généralement conclus pour une durée de cinq ans, sont des contrats dits à coûts bruts avec des bonus (qualité) et pénalités (en cas de non-respect des horaires).

Avec la soumission concurrentielle, les coûts de mise en œuvre du transport ont diminué de $30 \%{ }^{13}$ tout en maintenant le niveau de qualité. Parallèlement, les subsides opérationnels ont diminué d'environ $20 \%$. De plus, avec l'introduction de la concurrence, le niveau de prix du billet a diminué. On observe cependant une

11. SL Annual Report 2002.

12. Connex exploite le métro et une partie du réseau de bus.

13. YTV (2000). 
tendance à la concentration sur le marché ${ }^{14}$. En 2000, la plus grande partie des services étaient concentrés dans les mains de six entreprises, les petits opérateurs continuant à subsister en tant que sous-traitant. La procédure d'appel d'offres n'a pas changé depuis 1995 mais, dans les années à venir, les opérateurs devront accepter une part de responsabilité dans la profitabilité du service.

\subsection{Bruxelles}

À Bruxelles, le transport est géré par un opérateur public, la STIB, responsable des niveaux tactique et opérationnel. Les relations entre la STIB et l'AO, la Région de Bruxelles-Capitale (RBC), sont définies dans un contrat de gestion couvrant une période de cinq ans. Le contrat de gestion est peu incitatif et est principalement organisé sur la base d'une démarche veillant à promouvoir la qualité du service en octroyant un bonus lié aux performances. Sur le plan stratégique, la RBC définit les services de transport public nécessaires et finance les programmes d'investissement. Au point de vue tactique, la STIB est responsable de la stimulation de la demande et de l'amélioration de l'attractivité des services de transport public. La STIB a donc une certaine autonomie commerciale.

Le transport public à Bruxelles se caractérise par une organisation très peu concurrentielle. L'opérateur public exploite directement l'entièreté du réseau sans faire appel à la sous-traitance. II fait cependant face à une concurrence limitée sur certains tronçons de la part de deux autres opérateurs publics (TEC et DE LIJN). Finalement, la STIB se caractérise par des performances financières en dessous de la moyenne ${ }^{15}$. On observe toutefois une amélioration constante des performances opérationnelles et financières ces dernières années.

\section{CONCLUSIONS ET PERSPECTIVES}

Pour peu que le marché soit correctement encadré, l'introduction de mécanismes concurrentiels dans le transport urbain a un effet positif, à la fois sur la qualité du service et sur le niveau de subsides. Avec la généralisation de ces mécanismes au programme de la Commission européenne, se pose la question de savoir s'il est possible de maintenir une concurrence effective et durable dans le secteur du transport.

L'introduction de la concurrence a eu pour effet de modifier la taille des entreprises actives dans le secteur. À Stockholm comme à Helsinki, les petits opérateurs ont pratiquement disparu et, s'ils subsistent, ils n'opèrent quasi plus que comme sous-traitants de grandes compagnies nationales ou multinationales. Le processus de concentration du marché réduit l'efficacité du système d'appel d'offres, en particulier lorsque le service à concéder est complexe ${ }^{16}$. En France, où

14. Le nombre moyen d'offres reçues est passé de 7,6 à 4,5 entre 1994 et 2000 .

15. Berquin \& Wunsch (1997).

16. Yvrande-Billon (2004). 
l'entièreté du service est concédée à un opérateur unique, certaines AO ne reçoivent qu'une seule offre pour l'exploitation du service ${ }^{17}$. Diviser le réseau en plusieurs franchises à concéder est un moyen d'éviter la monopolisation du secteur, d'autant que la Commission européenne permet aux AO de tenir compte des parts de marché dans l'attribution des contrats ${ }^{18}$. La présence de plusieurs opérateurs permet également une évaluation comparative des coûts du service. La difficulté principale est cependant d'assurer la coordination de l'offre et l'intégration des services (horaires, billetterie, service clientèle) indispensables pour maintenir un réseau de qualité. C'est ce point qui a fait principalement défaut dans l'expérience de dérégulation anglaise.

À côté du maintien des petits opérateurs sur le marché, se pose la question du futur des opérateurs publics qui, pour certains, jouissent toujours d'une situation de quasi-monopole, comme c'est le cas à Bruxelles. Dans un marché concurrentiel, les opérateurs publics peuvent soit abandonner leurs responsabilités opérationnelles pour un rôle de planification et de coordination de l'offre (Stockholm), soit continuer d'offrir des services de transport. À Helsinki, les opérateurs publics sont présents sur les segments les plus complexes du marché (tram et métro) et, pour les services de bus, sur les segments où ils ont obtenu une franchise dans le cadre du processus d'appel d'offres. La possibilité de conserver un opérateur public sur certains segments du marché, voire sur l'ensemble de celuici, fait l'objet d'un intense débat. Pour les services de tram et de métro, le maintien d'un opérateur public, correctement régulé, se justifie pleinement par les compétences techniques des opérateurs en place, d'une part, et par la difficulté de mettre en place une concurrence effective pour ces services, d'autre part. Pour les services de bus, le recours à des mécanismes concurrentiels correctement encadrés permet d'accroître l'efficacité du service et d'utiliser plus efficacement les fonds publics. L'utilisation d'appels d'offres pour une partie des services présente le double avantage de pouvoir sélectionner l'opérateur le plus efficace et de mettre en place un processus de comparaison des coûts pour les différents opérateurs publics et privés. La procédure de gestion directe challengée devrait permettre de bénéficier également des avantages de la concurrence pour autant qu'il ne subsiste pas de barrières à l'entrée et que le marché soit effectivement contestable. Ce dernier point sera plus facilement acquis si les contrats sont de taille raisonnable. Si l'ensemble des services de transport est concédé directement à un seul opérateur, les possibilités de contester celui-ci seront relativement faibles.

Les cas étudiés dans cet article, tout en illustrant l'importance de la régulation dans le secteur du transport public, mettent en avant une problématique commune : la difficulté de mettre en place et de maintenir une concurrence effective. À ce titre, les autorités de la concurrence ont un rôle primordial : elles doivent garantir le bon fonctionnement des procédures d'appels d'offres et, dans un marché qui devient plus concentré, éviter l'émergence de pratiques concertées. Les AO, quant à elles, disposent de plusieurs moyens d'actions pour favoriser la mise en place

17. Commissariat général du Plan (2004).

18. L'article 9 de la proposition COM 2002107 permet à l'AO d'écarter l'offre d'un opérateur qui aurait (ou serait amené à avoir) plus de $25 \%$ de parts de marché. 
d'un marché concurrentiel. Par exemple, la procédure d'appel d'offres et les caractéristiques du service à concéder ont tous deux un impact sur le nombre d'offres reçues ${ }^{19}$. Outre l'utilisation de mécanismes de marché, les AO conservent une responsabilité importante dans la gestion des relations contractuelles avec les prestataires de services. La régulation des opérateurs est l'indispensable complément de la concurrence.

\section{BIBLIOGRAPHIE}

AB Storstockholms Lokaltrafik (SL), Annual Report 2002.

Berquin, P. et P. Wunsch (1997), Les transports en commun bruxellois en perspective, miméo.

CERTU/GART (2003), Transports collectifs urbains, enquête et analyse 2003.

Commissariat général du Plan (2004), L'économie et le financement des systèmes de transport urbains, Problèmes économiques, $n^{\circ} 2843,23-28$.

Commission des communautés européennes (2002), Amended proposal for a Regulation of the European Parliament and of the Council on action by Member States concerning public service requirements and the award of public service contracts in passenger transport by rail, road and inland waterway, COM 2002 107 Final.

Dalen, D.M. et A. Gomez-Lobo (2003), Yardsticks on the road : Regulatory contracts and cost efficiency in the Norwegian Bus Industry. Transportation, $\mathrm{n}^{\circ} 30,371$ 386.

European Metropolitan Transport Authorities (2003), EMTA News, $n^{\circ} 14$, septembre.

Gagnepain, P. (1998), Structure productive de l'industrie du transport urbain et effets des schémas réglementaires, Économie et prévisions, $n^{\circ}$ 135, 95-107.

Gagnepain, P. et M. Ivaldi (2002), Incentive Regulatory Policies: The Case of Public Transit Systems in France, Rand Journal of Economics, 33(4), 605-662.

Helsinki Metropolitan Area Council (YTV), Transportation in the Helsinki metropolitan area 2000.

Ivaldi, M., Jullien, B., Rey, P., Seabright, P. et J. Tirole (2003), The Economics of Tacit Collusion, Final report for DG Competition.

Klemperer, P. (2002), What Really Matters in Auction Design, Journal of Economic Perspectives, 16(1), 169-90.

Laffont, J.J. et J. Tirole (1986), Using Cost Observation to Regulate Firms, Journal of Political Economy, 94, 614-641.

Mackie, P., Preston, J. et C. Nash (1995), Bus Deregulation : Ten Years On, Transport Reviews, 15, 299-351.

Nash, C. (1993), British Bus Deregulation, The Economic Journal, 103, $1042-49$.

Yvrande-Billon, A. (2004), Les difficultés contractuelles de la délégation de services publics, Ecoflash n¹89.

19. Klemperer (2002). 